\title{
Three-Dimensional Architecture of the Endoneurium with Special Reference to the Collagen Fibril Arrangement in Relation to Nerve Fibers
}

\author{
Tatsuo Ushiki and Chizuka IDE \\ Department of Anatomy (Prof. C. IDE), Iwate Medical College School of Medicine, Morioka, Japan
}

Received October 13, 1986

Summary. The endoneurium of the mouse sciatic nerve was studied by scanning and transmission electron microscopy to elucidate their three-dimensional architecture.

The endoneurium consisted of collagen fibrils, and occasional fibroblasts and blood vessels. Collagen fibrils surrounded individual nerve fibers, forming two distinct layers of connective tissue sheath: the outer one was composed of bundles of longitudinally oriented collagen fibrils and the inner one was of a delicate network of interwoven thin collagen fibrils. These outer and inner layers of collagen fibrils correspond to the two fibrous sheaths known as the sheath of Key and Retzius and the sheath of Plenk and Laidlaw, respectively, revealed on nerve fibers by silver impregnation. Some of the finest collagen fibrils forming the inner network are closely attached to the basal lamina of Schwann cells, suggesting that these fibrils are concerned with the connection between the basal lamina and the inner collagen network. These two layers of collagen fibrils were found on all the nerve fibers, suggesting that they represent a general structure ensheathing the peripheral nerve fibers. Although these layers occur also on unmyelinated nerves, they are most developed on the largest myelinated fibers.

The endoneurium of the peripheral nerve is a connective tissue surrounding nerve fibers inside the perineurial sheath. The structure of the endoneurium was first described light microscopically by KEY and ReTzius (1873). They observed that each nerve fiber is wrapped by longitudinally oriented fibers called "Fibrillenscheide." Since then, this endoneurial sheath of collagen fibrils (sheath of Key and Retzius) has been studied and confirmed by several investigators such as RANvieR (1875) and CAJAL (1928) using mainly the silver impregnation method.

With improved silver impregnation methods, PLENK $(1927,1934)$ and LAIDLAW (1930) showed that there is a delicate network of intensely argyrophilic fine fibrils inside the sheath of Key and Retzius. This delicate argyrophilic network is now called the sheath of Plenk and Laidlaw. Based on Laidlaw's silver preparations, NAGeotTe (1932) presented a beautiful drawing of the sheath of Plenk and Laidlaw of a myelinated fiber in feline spinal nerve roots. However, classical methods of silver impregnation have the inherent difficulty of reliably demonstrating fine argyrophilic fibrils. Therefore, the fine network of argyrophilc fibrils of Plenk and Laidlaw has been since studied by few investigators (MAsson, 1932; HosoKawa, 1959). 
Studies by transmission electron microscopy (TEM) have revealed the fine structure of the endoneurium (CAusey and Barton, 1959; Thomas, 1963; Gamble, 1964; Gamble and Eames, 1964; Osawa and Ide, 1986). According to these studies, the endoneurium is composed mainly of longitudinally oriented collagen bundles, between which there are fixed or free connective tissue cells such as fibroblasts, macrophages and mast cells. These TEM studies of thin sections, however, provide little information about the three-dimensional arrangement of endoneurial collagen fibrils in relation to nerve fibers. In addition, no definite description has been made as yet concerning the fine structure of the collagen fibril network of the sheath of Plenk and Laidlaw.

Scanning electron microscopy (SEM) can provide an overall view of teased or cracked surfaces of tissues at the fine structural level. We applied SEM to the observation of the endoneurium in order to demonstrate the three-dimensional architecture of endoneurial collagen fibrils in relation to nerve fibers. The present study reveals the detailed structure of longitudinally oriented collagen bundles (the sheath of Key and Retzius), and also convincingly demonstrates the presence of fine collagen fibril meshworks lining the contour of nerve fibers as described by PLENK (1927) and LAIDLAW (1930).

\section{MATERIALS AND METHODS}

Adult mice (ddY strain) of both sexes, each weighing about $30 \mathrm{~g}$, were used in the present study. The animals were anesthetized by intraperitoneal injection of Nembutal (sodium pentobarbital $50 \mathrm{mg} / \mathrm{kg}$ body weight) and perfused via the left ventricle with a fixative containing $2 \%$ paraformaldehyde and $2.5 \%$ glutaraldehyde in $0.1 \mathrm{M}$ cacodylate buffer ( $\mathrm{pH}$ 7.4). Immediately after perfusion, the sciatic nerve was removed, cut into small segments and immersed in the same solution for more than $4 \mathrm{hrs}$ at room temperature.

For the SEM study, the epineurium and perineurium were carefully removed and the nerve was teased longitudinally into thin strips with forceps under a dissecting microscope. Some specimens were mounted on glass slides treated with $0.1 \%$ poly-Llysine solution. Both the mounted and non-mounted (free-floating) specimens were rinsed in a $1 \%$ aqueous solution of Tween 20 (polyoxyethylene sorbitan monolaurate) for several minutes, and then washed repeatedly with distilled water for more than one hour to clean their surfaces. The specimens were then treated with $2 \%$ tannic acid solution for 2-3 hrs, washed in distilled water for $1 \mathrm{hr}$ and immersed in $2 \%$ osmium tetroxide solution for several hours. They were then dehydrated through a graded series of ethanol. The non-mounted specimens were freeze-cracked in liquid nitrogen at the absolute ethanol stage during dehydration. After being transferred to isoamyl acetate, both the mounted and the non-mounted specimens were critical pointdried using liquid $\mathrm{CO}_{2}$, affixed on aluminum stubs, and coated with platinum in an ion coater (Eiko IB5, Eiko Engineering Co., Japan). Observations were made in an SEM (Hitachi HSM-2B) with an accelerating voltage of $10 \mathrm{kV}$.

For the TEM study, the specimens, fixed in the same manner as above, were then postfixed for $2 \mathrm{hrs}$ with $1 \%$ osmium tetroxide solution in $0.1 \mathrm{M}$ cacodylate buffer ( $\mathrm{pH}$ 7.4), dehydrated through a graded ethanol series and embedded in Epon 812. Ultrathin sections were stained with uranyl acetate and lead citrate, and observed in a TEM (JEM 100B). 


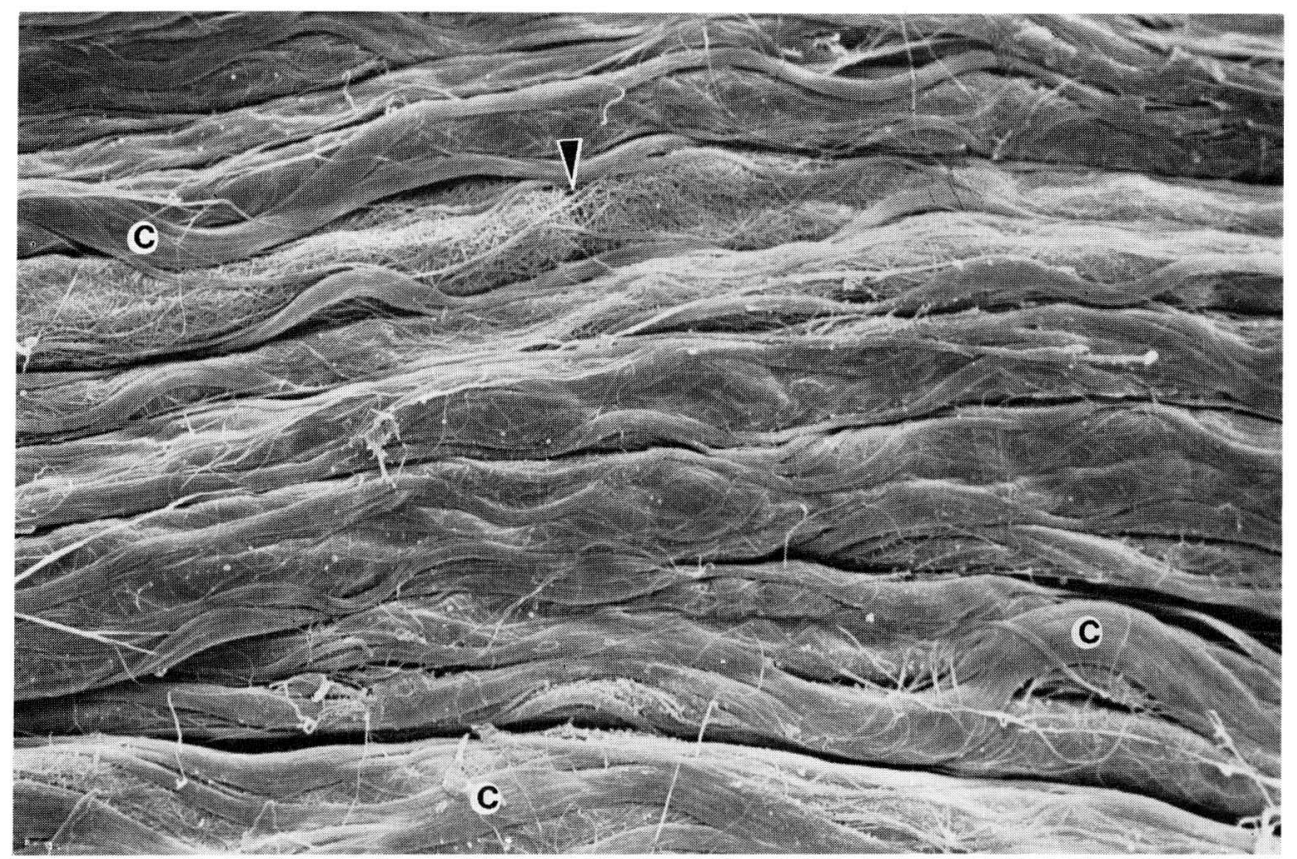

Fig. 1. Overview by SEM of the endoneurium of the mouse sciatic nerve. Bundles of collagen fibers (c) are oriented longitudinally on nerve fibers. Beneath these collagen bundles is seen a delicate network of fine collagen fibrils on some nerve fibers, which are especially evident in the region of node of Ranvier (arrowhead). $\quad \times 2,100$

\section{RESULTS}

SEM observations of the mouse sciatic nerve showed that the endoneurium was composed of collagen fibrils, and occasional fibroblasts and blood vessels. No elastic fibers were revealed by either SEM or TEM.

In an SEM survey, thick collagen fibers measuring approximately $2-3 \mu \mathrm{m}$ in width appeared to run in a longitudinal direction along the axis of nerve fibers (Fig. 1). These thick collagen fibers were bundles of collagen fibrils measuring $20-40 \mathrm{~nm}$ in diameter as seen in thin sections by TEM (Fig. 5). At a higher magnification, these bundles looked like tapes taking wavy courses on the surface of nerve fibers (Fig. 2, 3).

The relationship between collagen bundles and nerve fibers was visualized more precisely when the endoneurium had been appropriately dissociated (Fig. 4). Most of the longitudinally oriented collagen bundles ran undulatingly along individual nerve fibers for a very long distance without extending themselves across neighboring nerve fibers. Only a few thin collagen fibrils were occasionally extended between adjacent nerve fibers. The collagen bundles running in a wavy fashion often subdivided into smaller bundles of about $0.5 \mu \mathrm{m}$ or less in width, which were interconnected with each other during their courses, forming a very coarse network around individual nerve fibers (Fig. 4). These collagen bundles extended themselves across the nodes of Ranvier without any modification (Fig. 6).

Beneath the sheath of the very coarse network of thick collagen bundles (i.e., the sheath of Key and Retzius) was an elaborate network of fine collagen fibrils lining the 


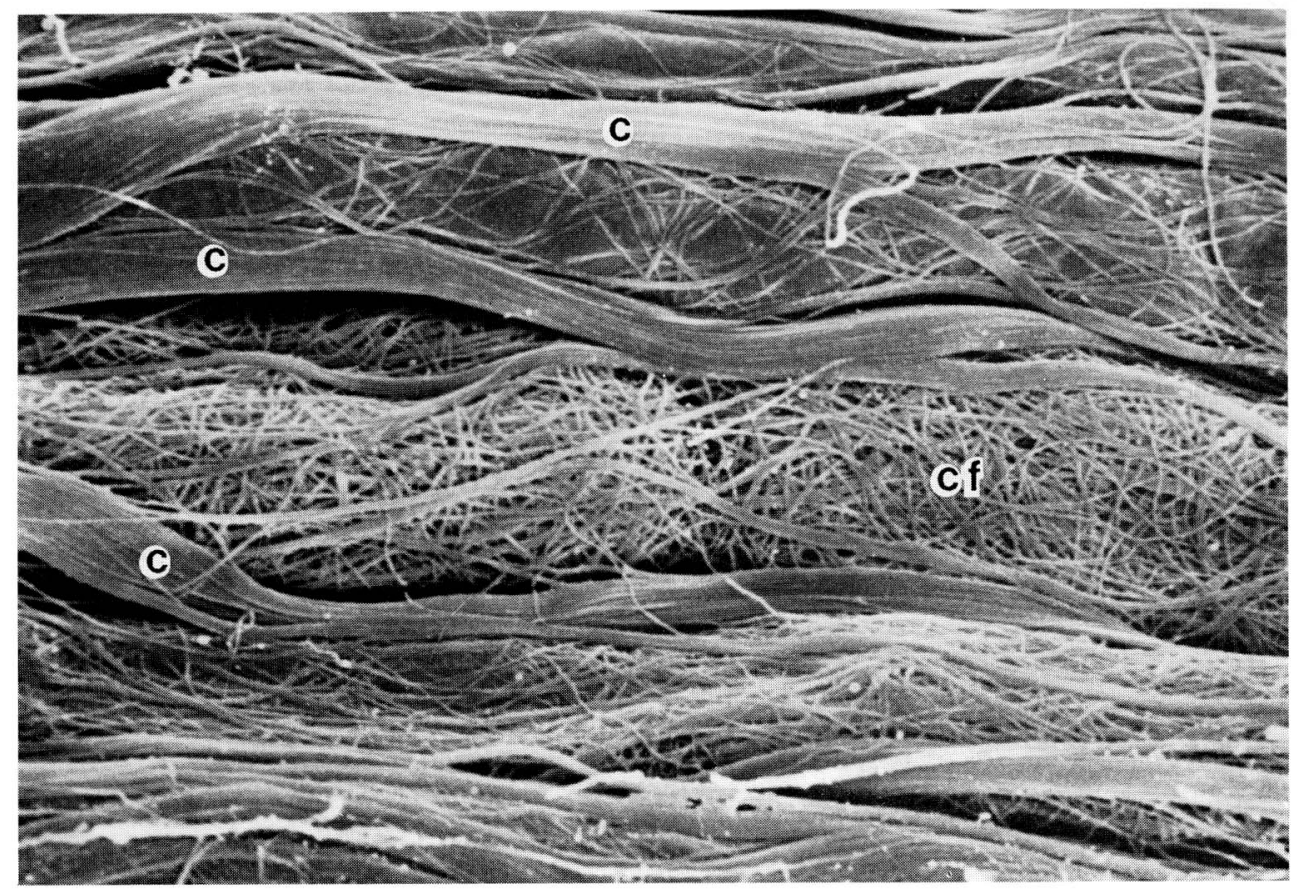

Fig. 2. Closer view of a part of Figure 1. Nodal and paranodal regions ensheathed by a dense meshwork of fine collagen fibrils $(c f)$. Other nerve fibers are enclosed at their internodal regions by a coarser network of fine collagen fibrils. $c$ Bundle of longitudinally oriented collagen fibers. $\times 5,300$

contour of nerve fibers in a continuous sheet (Fig. 2-4). These fine fibrils were oriented in every (circular, longitudinal and oblique) direction, forming a delicate meshwork. From TEM observation, it was evident that these elaborate collagen networks were composed of thin collagen fibrils measuring $20-40 \mathrm{~nm}$ in diameter (Fig. 5). These inner sheaths of fine collagen fibrils (i.e., the sheath of Plenk and Laidlaw) were found at varying degrees of development on all the nerve fibers. They were more developed on myelinated than on unmyelinated fibers, and there was a tendency towards network elaboration in proportion to the diameter of nerve fibers, the most conspicuous network occurring on the thickest myelinated fibers. In addition, on myelinated fibers the network was more elaborate and denser in the nodal and paranodal than in the internodal regions. These dense collagen networks in the nodal and paranodal regions were also clearly identified by TEM in the present study (Fig. 7). These inner sheath collagen meshworks often received branches of thin collagen fibrils (about 30-100 nm in diameter) from the longitudinal collagen bundles of the outer sheath. Such branches were especially abundant in the nodal and paranodal regions.

The interstitial sides of the basal lamina of Schwann cells could be identified by SEM. They exhibited a fairly smooth apearance on the whole (Fig. 8), but at a higher magnification appeared granular (Fig. 9). Some of the thinnest fibrils of the inner collagen networks, measuring approximately $30 \mathrm{~nm}$ in diameter (ca. $20 \mathrm{~nm}$ in thin sections), were closely attached to and contiguous with the interstitial surface of the Schwann cell basal lamina (Fig. 9).

There were a few blood vessels between nerve fibers. These were also ensheathed 


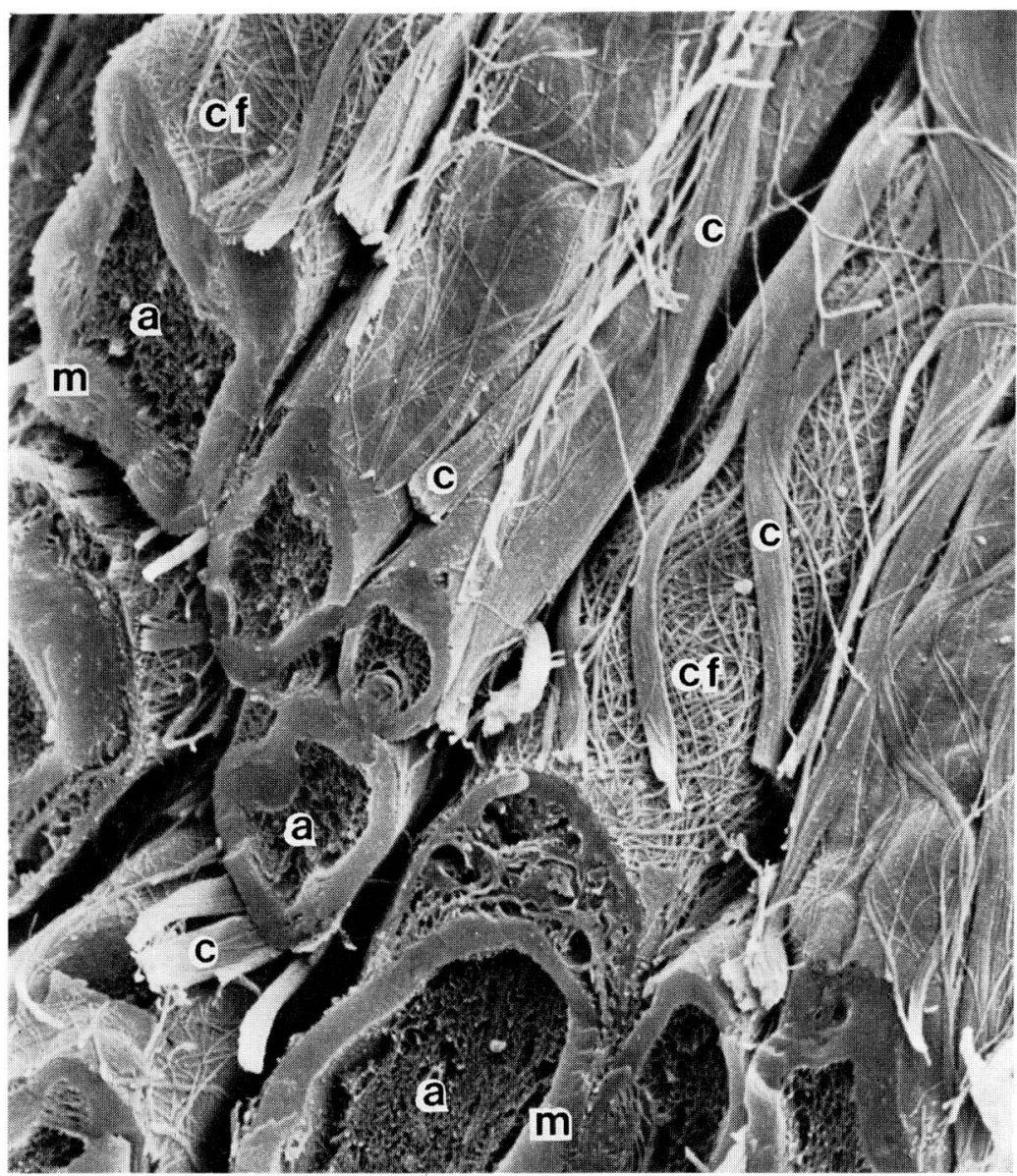

Fig. 3. Freeze-cracked portion of the sciatic nerve. Myelinated nerve fibers are cracked transversely. It is evident that both bundles of longitudinally oriented collagen fibers $(c)$ and underlying delicate networks of fine collagen fibrils $(c f)$ ensheath each nerve fiber. a Axon, $m$ myelin sheath $\times 4,700$

by networks of fine collagen fibrils similar to those on the nerve fibers. These meshworks of collagen fibrils (25-40 $\mathrm{nm}$ in diameter in thin sections) were, however, denser and more elaborately interwoven than those of nerve fibrers. Longitudinally oriented collagen fibers were rarely found on blood vessels (Micrograph not demonstrated).

Fibroblasts were occasionally observed by both SEM and TEM. They were slender and flattened in shape except for swellings at sites of nuclei, and had several laminar or wing-like processes extending between nerve fibers (Fig. 6, 7).

\section{DISCUSSION}

The present study revealed by SEM the three-dimensional architecture of the distinct outer and inner layers of collagen fibril sheaths of nerve fibers, which has been called the sheaths of Key and Retzius and of Plenk and Laidlaw in silver impregnation studies. 


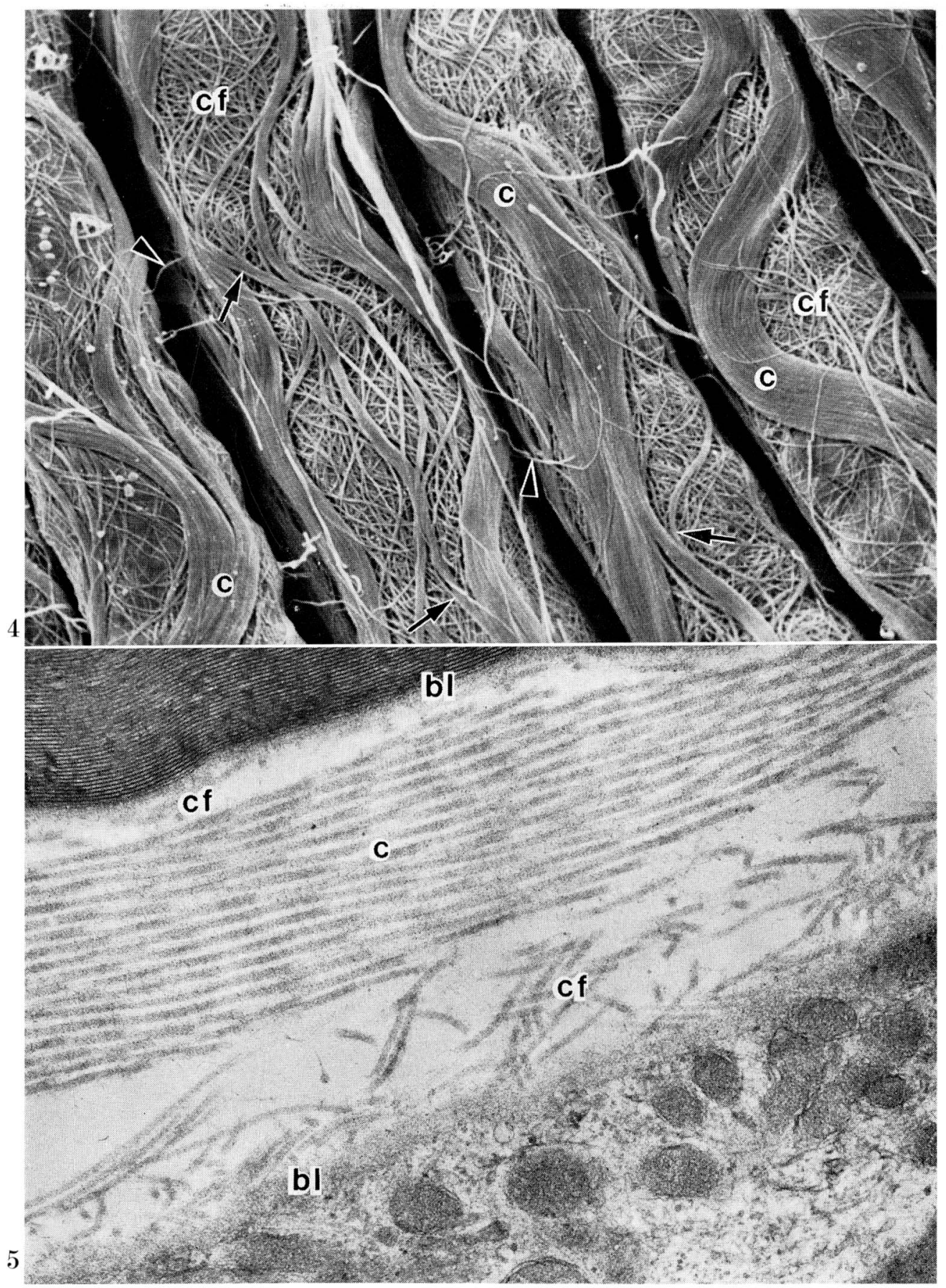

Fig. 4 and 5. Legends on the opposite page. 


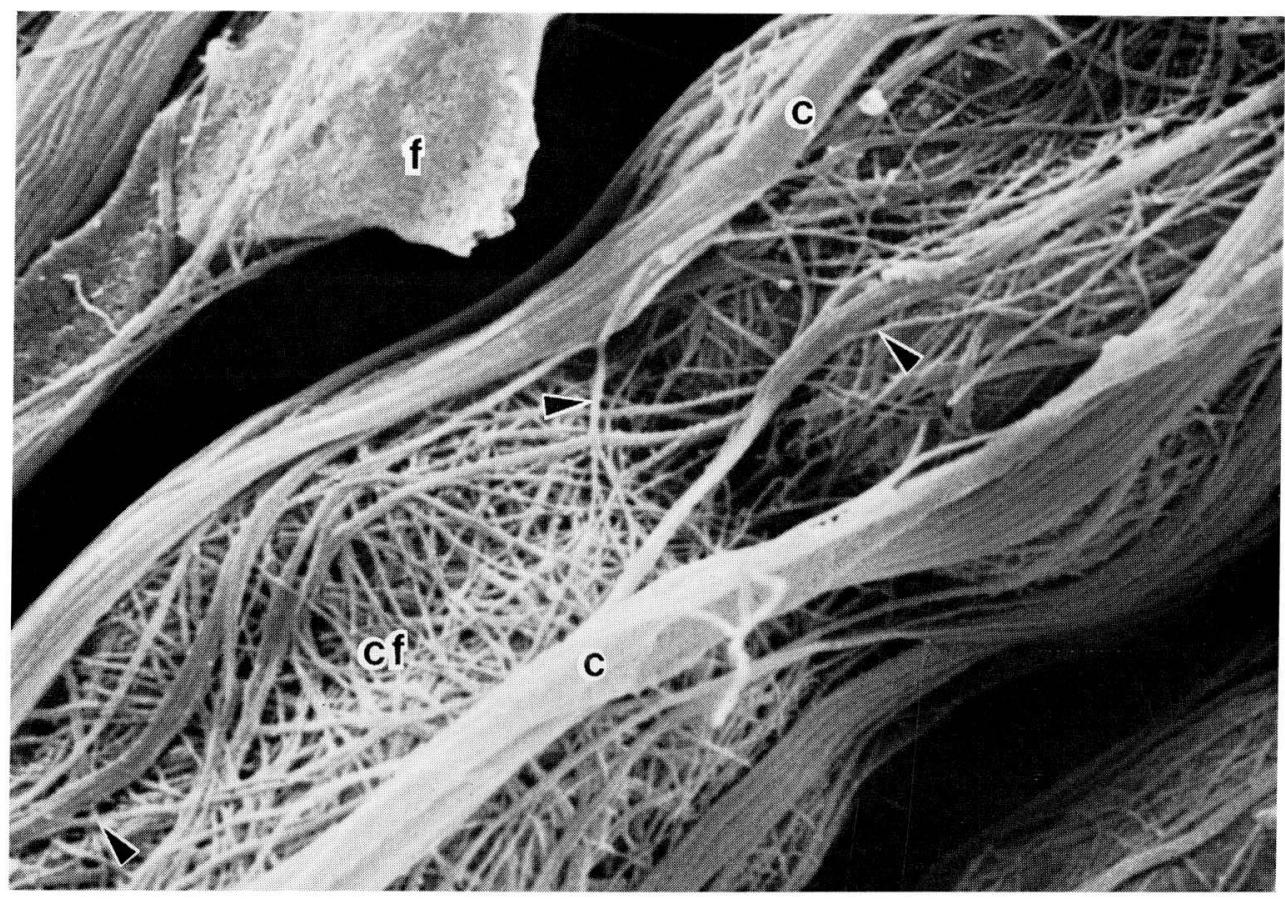

Fig. 6. Closer view of the nodal and paranodal regions of a myelinated nerve fiber. Collagen bundles (c) as well as an underlying thin collagen fibril network ( $c f$ ) span the node of Ranvier. The collagen fibril network appears to sink into the cleft of the node. Collagen bundles give rise to branches of fine fibrils (arrowheads), which partly contribute to forming the underlying fine collagen networks. $f$ Process of fibroblast. $\times 10,700$

Although several investigators (Spencer and Lieberman, 1971; Gershenbaum and Roisen, 1979; van Beek et al., 1979; Orgel and Huser, 1980) have observed peripheral nerves by SEM, no detailed information has been provided concerning the arrangement of endoneurial collagen fibrils. This would seem due to the presence of an amorphous ground substance which remained embedded the collagen fibrils in their specimens. In the present study we employed Tween 20 treatment and extensive washing of the specimens in successfully demonstrating the characteristic arrangement of endoneurial collagen fibrils in relation to nerve fibers.

\section{Connective tissue sheath around the nerve fibers}

It was Plenk $(1927,1934)$ who first recognized two layers of the endoneurial sheath enclosing individual myelinated fibers in human peripheral nerves by use of the silver impregnation method: the outer layer was composed of longitudinally oriented collagen

Fig. 4. Overview of myelinated nerve fibers which were slightly teased mechanically. Bundles of longitudinally oriented collagen fibers (c) often consist of smaller bundles (arrows), which are interconnected with each other during their courses. These collagen bundles and the underlying collagen fibril network $(c f)$ ensheath each nerve fiber. Neighboring nerve fibers are only very rarely interconnected by thin collagen fibrils (arrowheads). $\times 3,800$

Fig. 5. TEM image of a longitudinal section of the nerve. A bundle of collagen fibrils (c) is found between two myelinated fibers. Thicker or thinner layers of randomly oriented fine collagen fibrils ( $c f$ ) are visible beneath the collagen bundle, attaching themselves to the Schwann cell basal lamina $(b l) . \quad \times 34,000$ 


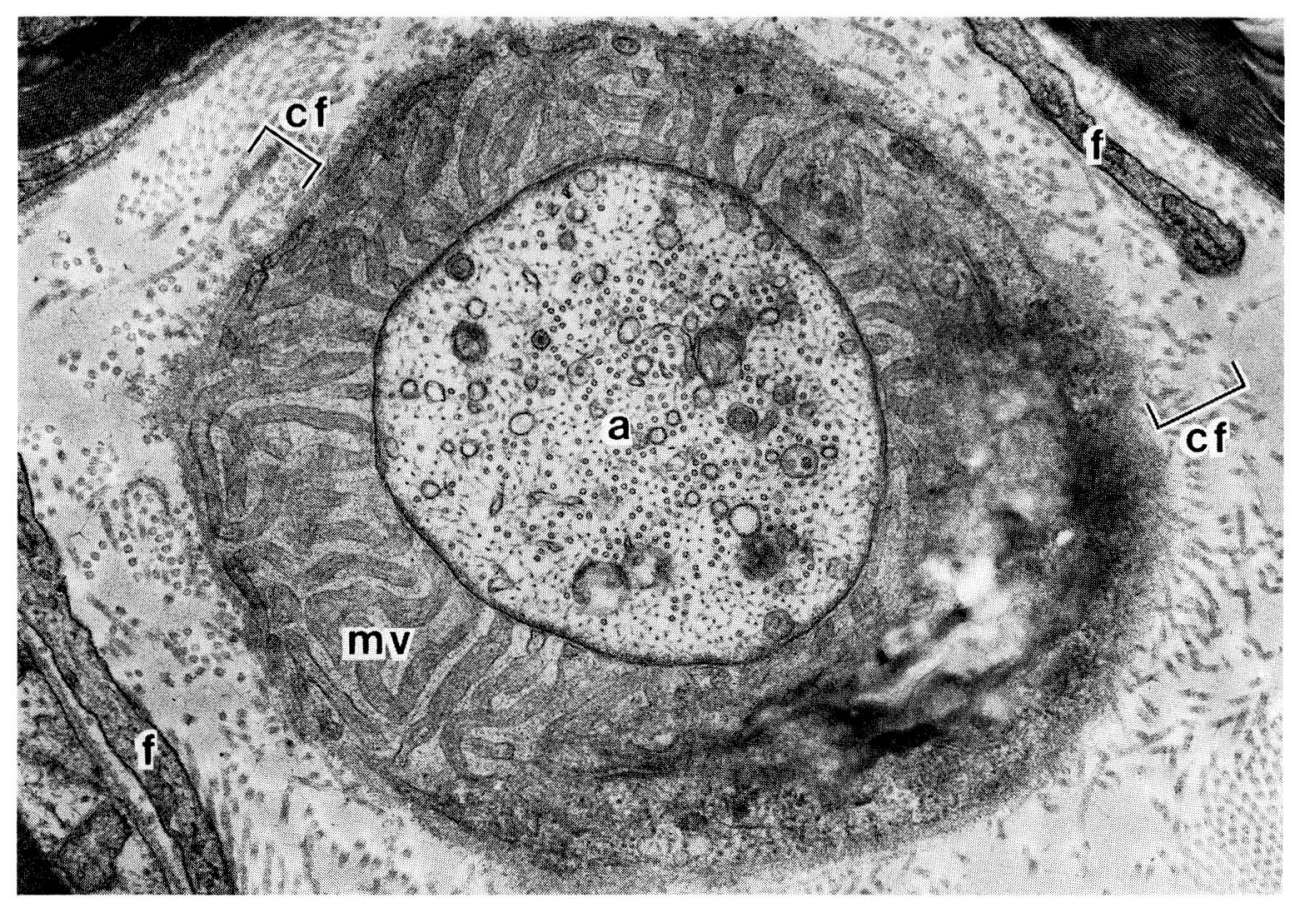

Fig. 7. TEM image of a transverse section through a node of Ranvier. A relatively thick layer of irregularly arranged collagen fibrils $(c f)$ is seen on the Schwann cell basal lamina of the node. $a$ Axon, $m v$ radial Schwann cell microvilli, $f$ fibroblast processes. $\times 22,500$

fibers, whereas the inner layer, of a delicate network of fine intensely argyrophilic fibrils. The same construction of the endoneurium was demonstrated more clearly by LAIDLAW (1930). The present study, by using modern methods, has confirmed and extended their light microscopic observations.

Modern TEM studies of thin sections have contributed but little toward demonstrating the three-dimensional arrangement of endoneurial collagen fibers. Only a few investigators ever noticed that the collagen fibers associated with nerve fibers are separable into two layers around large myelinated fibers: an outer densely packed layer of longitudinally oriented fibrils and an inner layer of irregularly oriented fibrils (rabbit limb nerve: Thomas, 1963; rat sciatic nerve: GAMBLE, 1964). They correctly pointed out that these layers corresponded to the sheath of Key and Retzius and that of Plenk and Laidlaw, respectively. However, they did not think that such layers of collagen fibrils were the general structure of peripheral nerve fibers. The reason for this is that when viewing small myelinated fibers in thin sections by TEM, the separation between the two layers is not definite. The present study used mouse sciatic nerves to demonstrate that the two layers of collagen fibrils are definitely present on all nerve

Fig. 8. Closer view of myelinated $(\mathrm{m})$ and unmyelinated $(\mathrm{um})$ fibers. The fine collagen network is less marked on the unmyelinated fibers. The interstitial aspect of the Schwann cell basal lamina is exposed beneath the collagen fibril meshwork. $c$ Bundle of collagen fibers, $c f$ delicate network of collagen fibrils. $\times 7,100$

Fig. 9. High magnification of a part of Figure 8. A small bundle of collagen fibers gives off branches of thin collagen fibrils (arrowheads). It appears that very thin collagen fibrils (arrows) are tightly attached to the Schwann cell basal lamina. The basal lamina was partially ablated (asterisk). $\times 21.000$ 


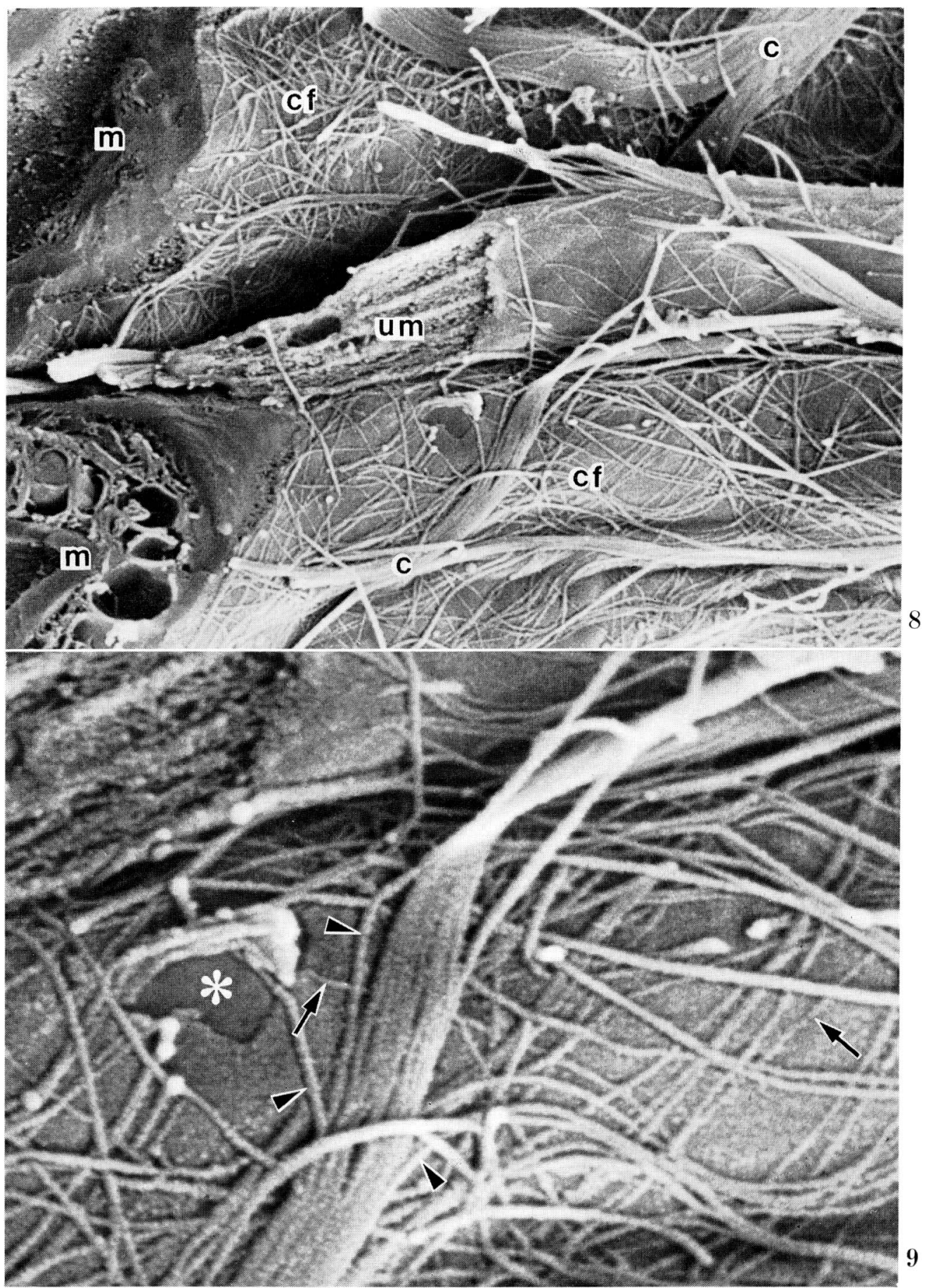

Fig. 8 and 9. Legends on the opposite page. 
fibers, including unmyelinated ones, although the inner collagen fibril network is less developed on smaller fibers. A similar arrangement of endoneurial collagen fibrils was also found by SEM on the fibers of rat and rabbit sciatic nerves (unpublished data). It is evident that these two layers of collagen fibril sheath are general structures on nerve fibers in the peripheral nervous system.

The longitudinally oriented collagen fibers may play an important role in resisting the stretching forces along nerve fibers, while the inner fine collagen meshworks may be concerned with protection against compression imposed on nerve fibers from the outside.

Laidlaw (1930) and Nageotte (1932) noted light microscopically that the inner layer receives branches of fine collagen fibrils from the outer longitudinal collagen fibers. Our SEM findings have confirmed such a continuity of collagen fibrils between these two layers. It can be said that these two layers as a whole form a basically continuous fibrous sheath on individual nerve fibers.

The present study has also revealed that the inner collagen network is denser in the nodal and paranodal than in the internodal regions, suggesting that solid protection by collagen fibril networks against mechanical distortions are needed at the nodal and paranodal regions.

\section{Collagen fibrils and the Schwann cell basal lamina}

Several TEM investigators have mentioned that collagen fibrils are thinner in the endoneurium than in the epineurium (Thomas, 1963; Gamble, 1964; JunqueIRA et al., 1979; OsAwA and IDE, 1986). In their TEM study of the mouse sciatic nerve, Osawa and IDE (1986) reported that collagen fibrils in the endoneurium were $25-30 \mathrm{~nm}$ in diameter. Our TEM observations coincide with their findings. In addition, the present study has revealed that collagen fibrils which are closely attached to the Schwann cell basal lamina are even thinner, measuring about $20 \mathrm{~nm}$ in diameter. Such a close attachment of very thin collagen fibrils to basal lamina has been reported by SAwADA (1981) by SEM in several tissues such as the exocrine pancreas and cardiac muscle. These extremely thin collagen fibrils may concern the connection between the basal lamina and the underlying collagen meshwork. Our combined light microscopic and SEM studies are making it more and more convincing that the layer of fine intensely argyrophilic fibrils surrounding epithelial structures, hitherto known as "light-microscopic basement membrane," are, under the electron microscope, a network of thin collagen fibrils closely attached to the basal lamina. Such a network and the basal lamina as a whole, form a distinct structural unit which demarcates and supports the epithelial elements (UsHiki, IsHII and IDE, unpublished). The present study suggests that a similar construction of collagen fibril network and basa lamina is also present on Schwann cells.

\section{REFERENCES}

Cajal, S. R.: Degeneration and regeneration of the nervous system. 1. (translated from Spanish) University Press, London, 1928.

Causey, G. and A. A. Barton: The cellular content of the endoneurium of peripheral nerve. Brain 82: 594-598 (1959). 
Gamble, H. J.: Comparative electron-microscopic observations on the connective tissues of a peripheral nerve and a spinal nerve root in the rat. J. Anat. (Lond.) 98: 17-25 (1964).

Gamble, H. J. and R. A. Eames: An electron microscope study of the connective tissues of human peripheral nerve. J. Anat. (Lond.) 98: 655-663 (1964).

Gershenbaum, M. R. and F. J. Roisen: A scanning electron microscopic study of peripheral nerve degeneration and regeneration. Neuroscience 3: 1241-1250 (1978).

Hosokawa, H.: Argyrophilic spiral fibers in the centro-peripheral boundary zone of the nervous system. J. comp. Neurol. 111: 407-425 (1959).

Junqueira, L. C. U., G. S. Montes and R. M. Krisztán : The collagen of the vertebrate peripheral nervous system. Cell Tiss. Res. 202: 453-460 (1979).

Key, A. and G. Retzius: Studien in der Anatomie des Nervensystems. Arch. mikrosk. Anat. 9: 308-386 (1873).

Laidlaw, G. F.: Silver staining of the endoneurial fibers of the cerebrospinal nerves. Amer J. Pathol. 6: 435-444 (1930).

Masson, P.: Experimental and spontaneous schwannomas (peripheral gliomas). Amer. J. Pathol. 8: 367-415 (1932).

Nageotte, J.: Sheaths of the peripheral nerves. Nerve degeneration and regeneration. In: (ed. by) W. Penfield: Cytology and cellular pathology of the nervous system. Hoeber, New York, 1932 (p. 189-239).

Orgel, M. G. and J. W. Huser : A comparison of light and scanning electron microscopy in nerve regeneration studies. Plast. Reconstr. Surg. 65: 628-634 (1980).

Osawa, T. and C. Ide: Changes in thickness of collagen fibrils in the endo- and epineurium of the mouse sciatic nerve during development. Acta anat. 125: 245-251 (1986).

Plenk, H.: Über argyrophile Fasern (Gitterfasern) und ihre Bildungszellen. Ergebn. Anat. Entw.Gesch. 27: 302-412 (1927).

: Die Schwannsche Scheide der markhaltigen Nervenfasern. Z. mikrosk.-anat. Forsch. 36: 191-214 (1934).

Ranvier, L.: Traité technique d'Histologie. Savy, Paris, 1875.

Sawada. H.: Structural variety of basement membranes: a scanning electron microscopic study. Biomed. Res. 2 (Suppl.): 125-128 (1981).

Spencer, P. S. and A. R. Lieberman: Scanning electron microscopy of isolated peripheral nerve fibers. Z. Zellforsch. 119: 534-551 (1971).

Thomas, P. K.: The connective tissue of peripheral nerve: an electron microscope study. J. Anat. (Lond.) 97: 35-44 (1963).

van Beek, A. L., S. C. Jacobs and E. G. Zook: Examination of peripheral nerves with the scanning electron microscope. Plast. Reconstr. Surg. 63: 509-519 (1979).

\author{
牛木辰男 \\ =020 盛岡市内丸 $19-1$ \\ 岩手医科大学医学部 \\ 解剖学第二講座
}

Dr. Tatsuo Ushiki

Department of Anatomy

Iwate Medical College School of Medicine

Morioka, 020 Japan 